\title{
Wavelet Daubechies (db4) Transform Assessment for WorldView-2 Images Fusion
}

\author{
Rubén Javier Medina-Daza*, Nelson Enrique Vera-Parra, Erika Upegui \\ Distrital University Francisco José de Caldas, Carrera 7 No. 40B - 53, Bogotá D.C., Colombia. \\ * Corresponding author. Tel.: +5713239300; email: rmedina@udistrital.edu.co \\ Manuscript submitted February 19, 2016; accepted April 18, 2016. \\ doi: $10.17706 /$ jcp.12.4.301-308
}

\begin{abstract}
This article implements a new Web service that offers users the possibility to merge images. Throughout the article three important issues are addressed. First, a Web service is implemented using free software to provide a simple interface for users to interact and request the image-fusion service. The second issue deals with the analysis of the Fast Haar Wavelet Transform (FHWT). The final part of the article is a description of the proposed methodology that actually performs image fusion using FHWT. Also in order to determine the efficiency of FHWT five different Wavelets were implemented in Matlab to merge the same pair of satellite images. By using four indices, the resulting images were evaluated in terms of both spatial quality and spectral quality. The best results of the evaluation process were obtained with FHWT, which preserves the spectral richness of the original multispectral image while improving spatial quality.
\end{abstract}

Key words: Daubechies, satellite images, image fusion, wavelet transforms.

\section{Introduction}

Conventional image fusion procedures are based upon several methods such as the RGH to HIS transformation, Brovey, Multiplication, Main Components among others. These methods are not completely satisfactory because they degrade the spectral information while gaining spatial information. This has promoted in the last years experiments of several procedures that use the Wavelet transform in two dimensions, since they degrade in less value the spectral richness of the original multispectral images [1]-[3] improving the spatial resolution. Based on research where is shown that the Wavelet transform improves the results of the satellite image fusion [4], the significant contribution of the Daubechies Wavelet transform for satellite image fusion allows to generate better fused images thanks to the way in which the resultant transform coefficients are arranged, thus obtaining the approximation and detail coefficients that contain higher spatial and spectral information from the original images. The fusion is an answer to the frequent need to process satellite images and the need to have in just one image high resolution spectral data (multispectral images) and high spatial resolution (panchromatic images) from the same sensor or from several remote sensors. With the fusion is obtained detailed information about the urban and rural environment, useful for several specific applications such as land management, agriculture, environmental management plans among others.

This work is focused in three main aspects: The first is the mathematical understanding of the Wavelet Daubechies transform. The second is about the description of the methodology for satellite image fusion using the Wavelet Daubechies transform. The third refers to the verification of its performance. The assessment is performed by comparing the performance of the Wavelet Daubechies (db4) transform with 
five decomposition levels [3], [5], [6] through four index, namely, the correlation coefficient, the RASE index, the ERGAS index and the universal quality index.

\section{Discrete Wavelet Transform}

Because of the complexity in the DWT numeric treatment, due to continuously variable scale parameters such as translation, it is essential to have a tool that allows to discretization of this. This is we are going to go from a continuous mapping to a spectrum or a set of finite values through the change of the integral by an approximation with summations. Discretization allows representing a signal in terms of elemental functions with coefficients.

The central idea of the wavelet transform analysis is to decompose a signal into different levels of resolution (for this research five levels will be used), this process is known as multiresolution [7]. The multiresolution representation provides a simple hierarchical framework for the interpretation of the information in the time series. At different resolutions, the details of a signal usually characterize different physical structures of themselves. At a coarser resolution, these details generally characterize large structures that provide information and with the increase of the resolution finer details are obtained [3]-[8].

\subsection{Wavelet Daubechies Function}

This type of wavelet is known as "compact support orthogonal wavelets", the name of the Daubechies family is writer as $\mathrm{dbN}$, where $\mathrm{N}$ is the order (or associated filter) and $\mathrm{db}$ is the short name of the wavelet. The wavelet db1 defines the same Haar wavelet [3], [6], [8].

For $N \in \mathbb{N}$, the Daubechies wavelet transform of class $D-2 N$ is a function $\psi={ }_{N} \psi \in L^{2}(\mathbb{R})$ defined as [4]:

$$
\psi(x):=\sqrt{2} \sum_{k=0}^{2 N-1}(-1)^{k} h_{2 N-1-k} \varphi(2 x-k)
$$

where $h_{0}, \ldots, h_{2 N-1} \in \mathbb{R}$ and those are the filter constant coefficients that satisfy the conditions below

$$
\sum_{k=0}^{N-1} h_{2 k}=\frac{1}{\sqrt{2}}=\sum_{k=0}^{N-1} h_{2 k+1}
$$

As well, for $l=0,1 \ldots, N-1$,

$$
\sum_{k=0}^{2 N-1+2 l} h_{k} h_{2 k-1}=\left\{\begin{array}{l}
1 \text { si } l=0 \\
0 \text { si } l \neq 0
\end{array}\right.
$$

where $\varphi={ }_{N} \varphi: \mathbb{R} \rightarrow \mathbb{R}$ is the scaling (Daubechies) function given by the recursion equation:

$$
\varphi(x):=\sqrt{2} \sum_{k=0}^{2 N-1} h_{k} \varphi(2 x-k)
$$

Obey to: $\varphi=0$ para $x \in \mathbb{R}\left([0,2 N-1]\right.$. As well for $\int_{\mathbb{R}} \varphi(2 x-k) \varphi(2 x-l) d x$ para $k \neq 1$.

The basic problem for the building of the Daubechies wavelets is the determination of the coefficients $h_{0}, \ldots, h_{2 N-1}$, that does not negate the scale function. It is to note that there are $N$ equations given by the orthonormality conditions. This return a total of $N+2$ equations for the $2 N$ filter coefficients $h_{k}$. Therefore, for $N=1$ is over determined, they are unique (if exist), and for $N>2$ they are undetermined. For the implementation of the Wavelet Daubechies transform, in this study [2], [9], [10], the db4 filter was used along with the ARSIS concept, the RGB-HIS transform and the Matlab software [3].

\subsection{The ARSIS Concept}


The "ARSIS Concept" (Accroissement de la Résolution Spatiale par Injection de Structures in french) is the increase of resolution by structure injection, this method uses the information with the higher resolution to inject it into the spectral resolution, which defines a strategy for MULTI images fusion with PAN images [11]. In the basic conditions of ARSIS there should be similarity between the physiographic structures observed in the spectral bands, without this implying an overlapping between bands neither the correlation coefficients among the images are high.

\section{Wavelet Daubechies Transform Implementation}

The Daubechies transform is applied computing the averages and differences through the scalar products with scale signals and wavelets, the only difference between them consist on how these scalar signals and wavelets are defined [5]. Coefficients $S(w)$ y $H(w)$ are symbols used for the multiresolution analysis. $H(w)$ is computed to guarantee that the orthonormality condition is met.

$$
\begin{gathered}
F(z)=\sqrt{\frac{1}{2(2-\sqrt{3})} \cdot(z-(2-\sqrt{3})) .} \\
F\left(e^{-i w}\right)=\sqrt{\frac{1}{2(2-\sqrt{3})}} \cdot\left(e^{-i w}-(2-\sqrt{3})\right) \\
H(w)=\left(\frac{1+\left(e^{-i w}\right.}{2} \cdot\right)^{2} \sqrt{\frac{1}{2}}\left(\frac{1}{\sqrt{2-\sqrt{3}}} \cdot\right)\left(e^{-i w}-(2-\sqrt{3})\right)
\end{gathered}
$$

The simpler number and the most used is the 2-D DWT Db4 which has four coefficients $h(0), h(1), h(2) \mathrm{y}$ $h(3)$, where:

$$
h(0)=\frac{(1+\sqrt{3})}{4 \sqrt{2}}=0,4830, h(1)=\frac{(3+\sqrt{3})}{4 \sqrt{2}}=0,8365, h(2)=\frac{(3-\sqrt{3})}{4 \sqrt{2}}=-0,1294, h(3)=\frac{(1-\sqrt{3})}{4 \sqrt{2}}=-0,1294
$$

The Daubechies scale function $y$ and the matrix built [6c]

$$
T=\left(\begin{array}{cccc}
h 0 & h 1 & h 2 & h 3 \\
h 2 & h 3 & h 0 & h 1 \\
h 3 & -h 2 & h 1 & -h 0 \\
h 1 & -h 0 & h 3 & -h 2
\end{array}\right)=\left(\begin{array}{cccc}
0,4830 & 0,8365 & 0,224 & -0,1294 \\
0,224 & -0,1294 & 0,4830 & 0,8365 \\
-0,1294 & -0,224 & 0,8365 & -0,4830 \\
0,8365 & -0,4830 & -0,1294 & -0,224
\end{array}\right)
$$

The complexity of the calculation is measured in terms of the number of multiplications and additions required to apply the transform. The efficient execution is a measure of how good is possible to perform the transform using parallel processing. That result is a simple algorithm and easy to use for a fast computation [7] suggest a fast algorithm to calculate the wavelet transform of a 2-D matrix, such method provides an orthogonal mixed matrix that gives advantages over the conventional methods in terms of calculations reduction and implementation simplification. To calculate a unique level of fast wavelet based on the orthogonal transform (FDWT) for the 2-D signal, the following steps must be followed:

1. The matrix " $X$ " must be the type $N \times N$, where $\mathrm{N}$ should be power of 2 .

2. A transformation matrix ( $T$ ) is built using the desired base train wavelets functions.

3. The transformation is applied by multiplying the transformation matrix $T$ by the input signal and multiplying the result for the $\mathrm{T}$ transposition as follows:

$Y=T . X . T^{\circ}$

where $Y$ is the $N \times N$ matrix, wavelet final transform of the input signal matrix $N \times N, X[6]$.

Matrix $X$ are arbitrary values, for this example is: 


$$
X=\left(\begin{array}{cccc}
120 & 25 & 71 & 100 \\
104 & 103 & 127 & 50 \\
160 & 139 & 110 & 147 \\
10 & 156 & 143 & 130
\end{array}\right)
$$

Transformation (Z), using the filter matrix of (T) coefficients:

$[Z]=[T][X]$. The $Z$ transposed matrix, $Z^{T}(d b 4)$,

Then the following operation is performed:

$[B]=[T][Z]^{T}$. The B transposed matrix

Finally the Matrix $(Y)$ must be equal to the transposed of the Matrix (B):

$[Y]=[T][X][T]^{T}, Y$ is db4

$$
Y=\left(\begin{array}{cccc}
197,1805 & 185,8252 & 23,6757 & 54,6356 \\
219,9877 & 244,5067 & -5,8259 & -74,9855 \\
47,8145 & 41,3642 & -44,6455 & 62,1998 \\
-67,5544 & 2,8757 & -31,0126 & 65,9583
\end{array}\right)
$$

To show the mathematical model of the Wavelet Daubechies transform and the multiresolution analysis, an example will be used from a $4 \times 4$ matrix that could represent a synthetic image or a satellite sub-image. Consider a simple function $f$ of size $(4 \times 4)$ a square matrix that meets the condition of being dyadic [2]. The complete wavelet transform of two dimensions can be written as:

$$
\left(\begin{array}{llll}
a_{11} & a_{12} & a_{13} & a_{14} \\
a_{21} & a_{22} & a_{23} & a_{24} \\
a_{31} & a_{32} & a_{33} & a_{34} \\
a_{41} & a_{42} & a_{43} & a_{44}
\end{array}\right) \underset{(T W d b N)}{\rightarrow}\left(\begin{array}{llll}
A 2 & H 2 & d_{12} & d_{14} \\
V 2 & D 2 & d_{32} & d_{34} \\
d_{21} & d_{23} & d_{22} & d_{24} \\
d_{41} & d_{43} & d_{42} & d_{44}
\end{array}\right)=\left(\begin{array}{ll}
A p 2 & c H 1 \\
c V 1 & c D 1
\end{array}\right)
$$

Thus the complete two-dimensional Wavelet Daubechies transform level two arises. All the coefficients from the left upper corners correspond to the approximation coefficients of second level, those are denoted as Ap2, where the coefficients H2, V2 and D2 are found. All the coefficients from the lower left corner correspond to the vertical detail coefficients of first decomposing level that is denoted as cV1. All the coefficients from the lower right corner correspond to the diagonal detail coefficients of first decomposing level, denoted as cD1. Ap2 is decomposed up to the five level.

\section{Methodology and Implementation of the Wavelet Daubechies 2-D Transform for Satellite Image Fusion}

\subsection{Study Zone}

The study area is located in Bogota (Colombia), specifically in the northwest sector. The zone of study is covered by a WorldView-2 sub image both panchromatic and multispectral. The sub panchromatic image has 0.5 meters of spatial resolution and it was taken on September $6^{\text {th }} 2007$, its reference system corresponds to UTM/WGS 84. The sub multispectral image has three channel (R-red, G-green and B-blue) and it has a spatial resolution of 2 meters, it was acquired on the same date as the panchromatic image and it has the same reference system. Both images where cropped to 4096 pixels width and 4096 pixels height to satisfy the Dyadic property [2], [4].

\subsection{Daubechies Wavelet Transform Implementation Method}

The implementation of the Daubechies Wavelet transform $\mathrm{db} 4$ for satellite image fusion is based on the 
process proposed [4] described above in the example which used the software Matlab. The fused images were generated up to the fifth level of decomposition.

As a result of this research the following steps are proposed:

Step 1. Register a color composition RGB (true color) of the multispectral image with the panchromatic image, using the same pixel size of this last one (0.5) meters. Transform the RGB image into HIS components (Hue, Intensity, Saturation).

Step 2. Apply the Daubechies Wavelet transform concept to the I component, iteratively up to the fifth level of decomposition [9], thus obtaining the following approximation and detail coefficients. cA5i approximation coefficients that contain the spectral information of the I component, cV1i, cH1i, cD1i, cV2i, cH2i, cD2i, cV3i, cH3i, cD3i, cV4i, cH4i, cD4i, cV5i, cH5i y cD5i detail coefficients where the spatial information of I is stored.

Step 3. Apply the Daubechies Wavelet transform concept to the panchromatic image up the to fifth level of decomposition obtaining in this way, and homologous to the previous step, the approximation and detail coefficients of the panchromatic image.

Step 4. Generate a new matrix concatenating the coefficients cA5i (they store the spectral information of the I component) and the spatial detail coefficients of fifth level of the panchromatic image cV5p, cH5p, cD5p, cV4p, cH4p, cD4p, cV3p, cH3p, cD3p, cV2p, cH2p, cD2p, cV1p, cH1p y cD1p.

Step 5. Apply the inverse Daubechies Wavelet transform to the obtained matrix in the previous step to obtain a the new intensity component (N-INT).

Step 6. Generate a new IHS (N-IHS) composition by joining N-INT along with the hue and saturation original components (obtained in step 1).

Step 6. Make the transformation from IHS to RGB, using the new composition N-IHS. In this way a new multispectral image is obtained which holds the spectral resolution thus gaining spatial resolution.

\section{Results}

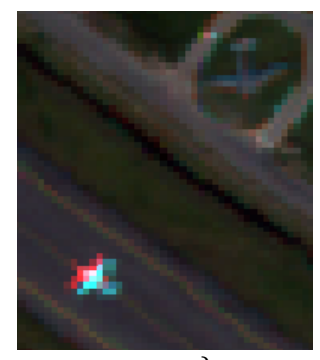

a)

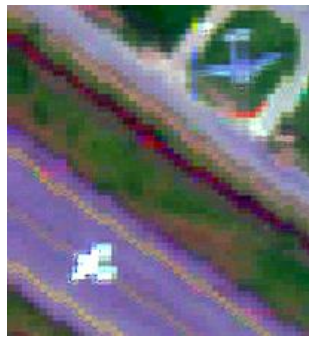

d)

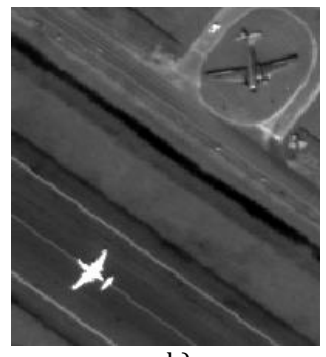

b)

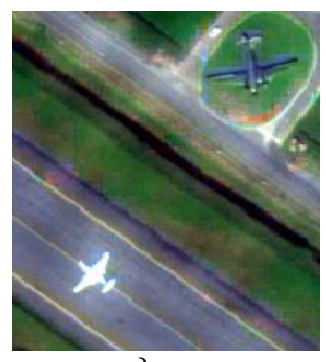

c)

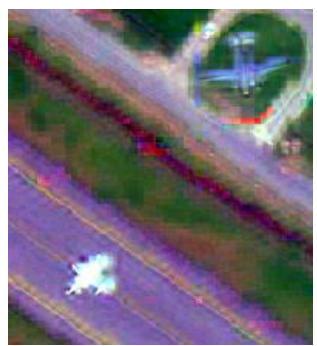

e)

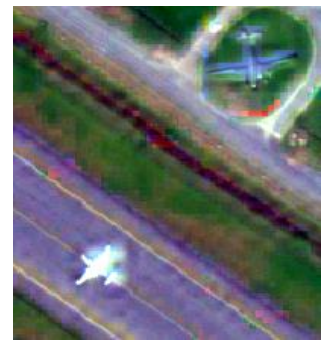

f)

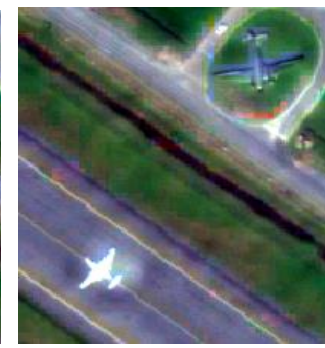

g)

Fig. 1. a) MULTI sub-Image detail. b) PAN sub-Image detail, c) fused sub-image db4-N5, d) fused sub-image with db4-N1, e) fused sub-image db4-N2, f) fused sub-image db4-N3, g) fused sub-image db4-N4.

Considering the implementation of the Daubechies Wavelet transform made in the five levels of decomposition N1, N2, N3, N4 and N5, five fused images are obtained (one for each level of decomposition), those were evaluated both qualitatively and quantitatively. 
For the assessment and the analysis of the results of the fused images using the Daubechies Wavelet, db4 predefined in Matlab, with five levels of decomposition, the following indexes were used: correlation coefficient (CC), RASE index, ERGAS index and the universal quality index $Q$, results are shown below:

\subsection{Correlation Coefficient (CC)}

The correlation between the different bands of the fused images and the bands of the original image is computed. In the Table 1 are shown the results of spatial and spectral correlation with the five chosen Wavelets from the Daubechies.

Table 1. Assessment of the WorldView-2 Image Quality Spatial and Spectral Correlation

\begin{tabular}{c|c|c|c|c|c|c}
\hline \hline \multirow{2}{*}{ Fused images by } & \multicolumn{3}{|c|}{ C.C. Spectral } & \multicolumn{3}{c}{ C.C. Spatial } \\
\cline { 2 - 8 } & R & G & B & R & G & B \\
\hline Db4-N1 & 0.93 & 0.98 & 0.94 & 0.65 & 0.86 & 0.59 \\
Db4-N2 & 0.92 & 0.97 & 0.92 & 0.65 & 0.88 & 0.59 \\
db4-N3 & 0.94 & 0.96 & 0.94 & 0.74 & 0.90 & 0.68 \\
Db4-N4 & 0.95 & 0.95 & 0.96 & 0.82 & 0.92 & 0.77 \\
Db4-N5 & 0.94 & 0.94 & 0.95 & 0.84 & 0.92 & 0.80 \\
\hline \hline
\end{tabular}

\subsection{ERGAS Index}

The definition of the relative global error dimensionless of synthesis called spectral ERGAS proposed by Wald [12] show the best results when it is closer to zero. On the other hand, Lillo and his team [13] propose another index called, ERGAS Espacial inspired by the spectral ERGAS index replacing MULTI for PAN, assessing in the same way the results. The assessment of the quality of the fused images done by these indexes is shown in Table 2.

\subsection{RASE Index}

The RASE index is expressed as a percentage [12] (see results on Table 2). The best results are obtained when the percentage is closer to zero.

\subsection{Universal Quality Index $Q$}

This model of index of quality identifies any distortion as a combination of three factors: correlation loss, luminance distortion and distortion contrast [14] (see results on Table 2).

The best values in this index are obtained when the value is closer to one.

Table 2. Assessment of the WorldView-2 sub Image Performed through the RASE, ERGAS Indexes and Universal Quality $Q$

\begin{tabular}{c|ccc|c}
\hline \hline Image fusion by & RASE & ERGAS Spectral & ERGAS Spatial & $Q$ \\
\hline db4-N1 & $54.71 \%$ & 12.96 & 135.98 & 0.83 \\
db4-N2 & $55.19 \%$ & 13.10 & 133.87 & 0.80 \\
db4-N3 & $34.96 \%$ & 8.44 & 103.53 & 0.85 \\
db4-N4 & $24.63 \%$ & 6.06 & 93.39 & 0.92 \\
db4-N5 & $24.94 \%$ & 6.18 & 93.50 & 0.92 \\
\hline \hline
\end{tabular}

Below is shown a brief analysis of the obtained results with the different indexes.

Regarding to the spectral correlation coefficient for the multispectral original images WorldView-2 with respect to the fused images, the higher value is obtained by the $\mathrm{G}$ band with a value of 0.98 (db4-N1), however globally the best average was obtained with the db4-N4 with a value of 0.953 . As for the spatial correlation between the WorldView-2 panchromatic image and each of the fused images bands, the best are achieved with db4-N5 and db4-N4, with averages of 0.855 y 0.836 respectively. Regarding to RASE, ERGAS spectral indexes and the quality index $Q$, the best results of the image fusion with the WorldView-2 image 
are achieved with db4-N4 being $24.63 \%, 6.06$, and 0.92 , respectively. Regarding to the ERGAS spatial index, the same as the previous indexes the higher values are achieved with db4-N4 reaching a value of 93.39. This way, the best results of the assessment were obtained with the proposed method db4-N4 as they are in the optimum range showing that the fused images preserve the spectral richness of the multispectral original image and its spatial quality is improved.

\section{Conclusion}

The research previously presented have shown that the fusion methods of images based on the Wavelet transform are more suitable for the fusion of images than conventional methods. The Daubechies (db4) Wavelet transform, decomposing level four ( $\mathrm{N}-4)$ provided the best results at the time to fuse satellite images. This way the fused images provide to the users detailed information about the urban and rural environments which is useful for applications as planning and urban management. It usefulness extends to the development of projects in several fields as agriculture, hydrology, environment and emergency management caused by natural disasters (floods, forest fires), among others.

\section{References}

[1] Burrus, C. S., Gopinath, R. A., \& Guo, H. (1998). Introduction to Wavelets and Wavelet Transforms. Prentice-Hall.

[2] Nievergelt, Y. (1999). Wavelets Made Easy, Birkhäuser, Boston.

[3] Misiti, M., Misiti, Y., Oppenheim G., \& Poggi, J-M. (1996). Wavelets Toolbox for use with MATLAB, The Maht works Inc.

[4] Nuñez, J. O., Fors, X., Prades, O., Pala, A., \& Arbiol, R. V. (1999). Multiresolution-based image fusion whit additive wavelet descomposition. IEEE Transactions on Geoscience and Remote Sensing, 37(3), 1204-1211.

[5] Mallat, S. (1996). Wavelets for a vision. Proceedings of the IEEE: Vol. 84 (pp. 604-614).

[6] Mahmoud, W. A., Ahmed, S. H., \& Talib, M. J. (2012). Development of a 2-D wavelet transform based on Kronecker product. University of Baghdad, 15(4), 8-13.

[7] Mahmoud, M., \& Dessouh, M. (2007). Comparison between Haar and Daubechies Wavelet transfonnations on FP_GA technology. World Academy of Sc1ence, Engineering, and Technology, 26, 68-72.

[8] Medina, J., \& Pinilla, C. (2010). Algoritmos Matemático para la fusión de imágenes satelitales. V Simposio Internacional de Sistemas de Información e Ingeniería de Software en la Sociedad del Conocimiento.

[9] Medina, J., Pinilla, C., \& Joyanes, L. (2013). Two-dimensional fast Haar wavelet transform for satellite-image fusion. Journal of Applied Remote Sensing, 7(1).

[10] Medina, J., \& Lizarazo, I. (2004). Fusión de imágenes satelitales usando la transformada de Wavelet. Bogotá: Universidad Distrital Francisco José de Caldas.

[11] Ranchin, T., \& Wald, L. (2000). Fusion of high spatial and spectral resolution images: The ARSIS concept and its implementation. Photogrammetric Engineering \& Remote Sensing, 66(1), 49-61.

[12] Wald, L. (2002). Data fusion, definition and architectures. Fusion of Image of Different Spatial Resolution, Paris: Le Presses de l'École des Mines.

[13] Lillo-Saavedra, M. G., Arquero, A. C., \& Martinez, E. (2005). Fusion of multispectral and panchromatic satellite sensor imagery based on tailored filtering in the Fourier domain. International Journal of Remote Sensing, 26, 1263-1268.

[14] Wang, Z., \& Bovink, A. C. (2002). A universal image quality index. IEEE Signal Processing Letters. 


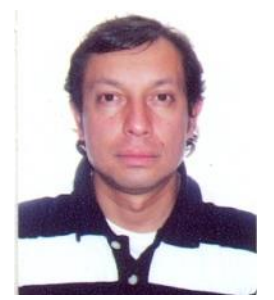

Rubén Medina Javier Daza has a PhD in computer Science with emphasis in geographic information systems (GIS) from Pontifical University of Salamanca Campus Madrid (UPSAM) in 2013. He received his master's degree in teleinformatics from District University Francisco José de Caldas (UDFJC), Bogotá, Colombia in 2002, other degrees include Specialist in software engineering in 1997, specialist in GIS in 2005 and mathematics from UDFJC in 1995.

$\mathrm{He}$ is currently a Titular Professor at UDFJC, working for the Faculty of Engineering (Geodesy and Cadastral Engineering) and for the Master of Science in Information and Communications program. Dr. Medina graduated Cum laude from UPSAM, some of his publications includes: Two-dimensional fast Haar wavelet transform for satellite-image fusion (USA, Journal Of Applied Remote Sensing, 2013), Aplicativo Web para la Fusión de Imágenes Satelitales (Oporto, Portugal: Risti - Revista Ibérica De Sistemas E Tecnologias De Informação, 2013), Matemáticas especiales. Una aplicación de la transformada de Fourier en el análisis de imágenes (Bogotá, Colombia: District University Editorial, 2013). He is currently researching in mathematical computing.

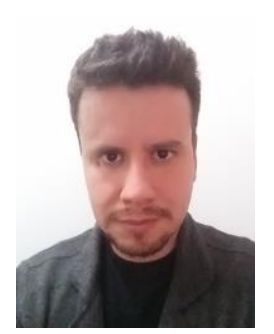

Nelson Enrique Vera Parra received his master in information science and communications from District University Francisco José de Caldas (UDFJC), Bogotá, Colombia in 2008. He is an electronic engineer from the South Colombian University, Neiva, Colombia from 2002. He is also a PhD student in UDFJC.

He is currently a Titular Professor at UDFJC, working for the Faculty of Engineering (Electronic Engineering). Some of his publication includes biopython basic, practice manual (Bogotá, Colombia: District University Editorial, 2014), Optimización del preprocesamiento de lecturas de secuenciación de nueva generación (Bogotá, Colombia: Redes De Ingeniería, 2014), the immunotranscriptome of the Caribbean reef-building coral Pseudodiploria strigosa (Germany, Inmunogenetics, 2015). He is currently researching in genome assembly processes and heterogeneous computing.

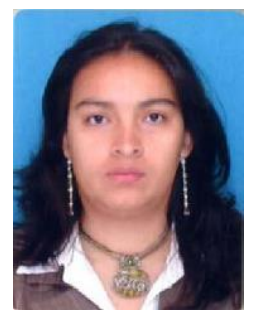

Erika Upegui was born in Puerto Boyacá (Colombia), she obtained a PhD in geography and spatial planning of the University Franche-Comté (Besançon, France) in 2012. She made her magister in remote sensing and geomatics applied to the environment at the University of Paris 7 (Paris, France), in 2009. Other studies include specializations on appraisals in 2005 and geographical information systems in 2002 and cadastral engineering and geodesy in 2002 at UDFJC.

She has worked in public and private industry sector companies, has become part of interdisciplinary working groups, had a doctoral contract for the execution of her doctoral work financed by Region Franche-Comté (France) and since 2013 is a full time Professor at Universidad Distrital Francisco José de Caldas. For a year she was the coordinator of the Cadastral Engineering and Geodesy faculty.

Dr. Upegui is a member of the SELPER chapter Colombia and she is a member of IEEE, she won the award of young researchers of the region Franche-Comté "Prix A'Doc" 2012, her main publications are in the remote sensing area and public health, working particularly in the estimation of exposed populations (Photogrammetric Engineering and Remote Sensing, 2012), the impact of heat waves (International Journal of Health Geographics, 2012), the estimation of concentrations of cyanobacteria using satellite images(Environment international, 2015). 